\title{
Prevalence of human papillomavirus among oesophageal squamous cell carcinoma cases: systematic review and meta-analysis
}

\author{
J L Petrick ${ }^{*, 1,5}$, A B Wyss ${ }^{1,2,5}$, A M Butler ${ }^{1,5}$, C Cummings ${ }^{1}$, X Sun ${ }^{1}$, C Poole ${ }^{1}$, J S Smith ${ }^{1,3}$ and A F Olshan ${ }^{1,3,4}$ \\ ${ }^{1}$ Department of Epidemiology, Gillings School of Global Public Health, University of North Carolina, Chapel Hill, NC 27599, USA; \\ ${ }^{2}$ Epidemiology Branch, National Institute of Environmental Health Sciences, National Institutes of Health, US Department \\ of Health and Human Services, Research Triangle Park, NC 27709, USA; ${ }^{3}$ Lineberger Comprehensive Cancer Center, School of \\ Medicine, University of North Carolina, Chapel Hill, NC 27599, USA and ${ }^{4}$ Otolaryngology/Head and Neck Surgery, School of \\ Medicine, University of North Carolina, Chapel Hill, NC 27599, USA
}

Background: Oncogenic human papillomavirus (HPV) has been hypothesised as a risk factor for oesophageal squamous cell carcinoma (OSCC), but aetiological research has been limited by the varying methodology used for establishing HPV prevalence. The aims of this systematic review and meta-analysis were to estimate the prevalence of HPV DNA detected in OSCC tumours and the influence of study characteristics.

Methods: Study-level estimates of overall and type-specific HPV prevalence were meta-analysed to obtain random-effects summary estimates.

Results: This analysis included 124 studies with a total of 13832 OSCC cases. The average HPV prevalence ( $95 \%$ confidence interval) among OSCC cases was $0.277(0.234,0.320)$ by polymerase chain reaction; $0.243(0.159,0.326)$ by in situ hybridisation; $0.304(0.185$, 0.423 ) by immunohistochemistry; $0.322(0.154,0.490)$ by L1 serology; and $0.176(0.061,0.292)$ by Southern/slot/dot blot. The highest HPV prevalence was found in Africa and Asia, notably among Chinese studies from provinces with high OSCC incidence rates.

Conclusions: Future research should focus on quantifying HPV in OSCC cases using strict quality control measures, as well as determining the association between HPV and OSCC incidence by conducting large, population-based case-control studies. Such studies will provide a richer understanding of the role of HPV in OSCC aetiology.

Oesophageal cancer is the eighth most common cancer worldwide, with an estimated 481000 annual incident cases, and is the sixth most common cause of cancer mortality, with an estimated 406000 annual deaths (Ferlay et al, 2010). Incidence rates of oesophageal cancer vary greatly by geographic region (Corley and Buffler, 2001; Ferlay et al, 2010), with markedly high rates in the 'Asian Oesophageal Cancer Belt', which extends from northern Iran, east to China and north into Russia (Glenn, 2001; Kamangar et al, 2007; Wei et al, 2011). Reasons for the geographic variability are not well understood (Hongo et al, 2009).
Squamous cell carcinoma is the most common form of oesophageal cancer (Corley and Buffler, 2001). Epidemiological studies conducted in the Western countries, Asia, and Africa have concluded that tobacco and alcohol consumption are the main known risk factors for OSCC (IARC, 1986; IARC, 1988; Munoz and Castellsague, 1994), accounting for approximately $90 \%$ of OSCC in men and 29\% in women (Castellsague et al, 1999). Yet, it has been suggested that alcohol and tobacco consumption are not the major risk factors for OSCC in one of the highest risk areas of northern China (Linxian County) (Yu et al, 1993). Additional

\footnotetext{
*Correspondence: JL Petrick; E-mail: jessica.petrick@unc.edu

${ }^{5}$ These authors should be regarded as joint First Authors.
}

Received 15 November 2013; revised 17 January 2014; accepted 28 January 2014; published online 11 March 2014 
established risk factors include poverty and nutritional deficiencies (Hongo et al, 2009; Mao et al, 2011).

Infection with oncogenic HPV as a contributor to OSCC was hypothesised over three decades ago (Syrjanen, 1982). However, the International Agency for Cancer Research (IARC) has concluded that there is inadequate evidence in humans for HPV carcinogenicity in association with OSCC (IARC, 2012).

The aims of this systematic review and meta-analysis were to estimate the prevalence of HPV detected in OSCC cases and the influence of type of HPV detection methods and other study characteristics on this measure.

\section{MATERIALS AND METHODS}

Search strategy and inclusion criteria. A systematic literature search was conducted using MEDLINE (via PubMed) and Excerpta Medica database (EMBASE) through 12 July 2013, with no specified start date or language restrictions. This systematic review was conducted in accordance with PRISMA guidelines (Moher et al, 2009).

Broad search terms included: HPV, oesophagus, and cancer (e.g., neoplasm, carcinoma, tumour) (specific search terms available upon request). Eligible studies included peer-reviewed publications with HPV prevalence data from a minimum of 20 adult human cases of primary, invasive, and oesophageal squamous cell carcinoma (OSCC). Review articles and editorials were included if they contained original data. Abstracts were excluded. Primary and secondary references were searched to identify additional studies. A medical reference librarian verified the search strategy, and independently repeated the search. If multiple publications presented data from the same population, only the publication with the largest sample size was included. Articles written in languages other than English were translated and reviewed. Because articles were excluded based on multiple criteria, we assigned a primary exclusion criterion based on the following rank-order: study not conducted in humans (i.e., in vitro or animal study), not oesophageal cancer, histology other than squamous cell carcinoma, not primary tumour, not invasive cancer (i.e., in situ only), study population included only children (i.e., $<18$ years old), letter to the editor or review (without original data), case report, abstract, histology unidentified, $<20$ cases, and no quantification of HPV.

Data extraction. For studies meeting our inclusion criteria, the following data were extracted: first author, publication year, study location, dates of sample collection, sample size, specimen type, HPV detection method, and overall and type-specific prevalence of HPV. Continent, country, and region were recorded. For studies with samples from multiple countries, data were extracted separately by country when possible.

Studies were categorised into five geographical areas: Africa, Asia, Europe and Australia, North America, or South and Central America. In addition, we analysed separately the studies performed in Iran and the high-OSCC-incidence province-level divisions of China (i.e., provinces with higher than the overall crude incidence of oesophageal cancer for China of 22.1/100000) (Chen et al, 2013). These included Fujian, Gansu, Hebei, Henan, Jiangsu, Shandong, Shanxi, and Sichuan provinces, as well as Xinjiang Uyghar Autonomous Region.

Specimen type was classified as paraffin-embedded fixed biopsies, fresh or frozen biopsies, or blood samples. HPV detection method was classified as polymerase chain reaction (PCR), in situ hybridisation (ISH), immunohistochemistry, serology, and Southern blot (including dot and slot blot). For studies that used multiple HPV detection methods, overall and type-specific prevalence of HPV were extracted independently. For studies that utilised PCR, information on specific HPV primers used was also extracted. For studies that reported use of multiple PCR primers, only the three most sensitive primers were recorded. The following rank order of sensitivity (from most to least sensitive), based on a meta-analysis of invasive cervical cancer, was assumed: SPF10, GP5 + /6 +, L1C1/2, MY09/11, PU1M/2R, GP5/6, L1, E6, E7, HPV-16 specific, and HPV-18 specific (Clifford et al, 2003). HPVtype-specific estimates were only recorded if the authors reported which HPV types were tested. For studies that utilised serology, data on HPV prevalence were extracted separately by serological marker (i.e., L1 vs E6/E7).

Data extraction was performed for each article by two of five independent reviewers (AMB, ABW, CC, JLP, XS) using a standardized data extraction form, and discrepancies were resolved by consensus. Study authors were contacted to obtain specific needed information. This occurred when the reviewers were unable to determine 1) HPV prevalence by detection method or 2) HPV prevalence by HPV subtype.

Statistical analysis. For each study, overall HPV prevalence estimates were calculated as the percentage of OSCC cases who tested positive for any HPV type. In some studies, the total number of available OSCC cases differed from the number of OSCC cases actually tested for HPV (e.g., cases were not tested if they were beta-globin-negative). Additionally, if one HPV detection method was utilised as a screening method for another, we did not include the results of the secondary screening method in our analyses. Information on all HPV-based studies is presented in Supplementary Table 1. The number of OSCC cases tested was used as the denominator. For studies reporting type-specific HPV prevalence, estimates were calculated as percentages of OSCC cases (same denominator as for overall prevalence) that tested positive for a single type of HPV. Infections for multiple types of HPV were separated into types; thus, type-specific prevalence represents types for tumours with either single or multiple infections. Data on overall and type-specific HPV prevalence are presented for each study in the appendices by HPV detection method. We calculated the variance of each prevalence estimate as $p q / n$, where $p$ is the prevalence, $q$ is $1-p$, and $n$ is the number of OSCC cases (Barendregt et al, 2013).

For studies reporting HPV prevalence equal to zero we used an empirical continuity correction method described by Sweeting et al (2004) to smooth the zero values. Specifically, we estimated the pooled prevalence, by detection method, for studies with non-zero prevalence estimates. These estimates divided by 100 were then used as the number of HPV-positive OSCC cases, and 1 minus the value added to the HPV-positive OSCC cases was added to the number of HPV-negative OSCC cases. A similar approach was used to smooth HPV type-specific estimates in studies that explicitly reported zero OSCC cases that were positive for a particular HPV type (e.g., 0 OSCC cases who were HPV-16 positive). If studies did not explicitly report type-specific HPV prevalence, this information was considered missing and not smoothed.

Two studies (Ma et al, 2000; Sitas et al, 2012) provided only type-specific HPV prevalence estimates, and overall HPV prevalence estimates could not be determined (i.e., unable to reconcile single $v s$ multiple type infections). In such instances, the overall HPV prevalence was considered to be missing and was not smoothed but these studies were included in the type-specific analyses.

For the analyses, we used three models: (1) crude model, (2) univariable model, and (3) multivariable model. The crude model was run by HPV detection type for overall prevalence of HPV (Supplementary Table 2). The univariable model was used to explore the heterogeneity of overall random-effects estimates (Table 2). Both overall (Table 2) and prevalence estimates stratified by HPV detection type (Supplementary Table 2) were considered 
from models that included HPV prevalence and disjoint indicator variables for a single study characteristic that included: geographic location, publication date, DNA source, and primer set (for PCR only). For example, running an overall HPV prevalence model for HPV detection type would include HPV prevalence and a disjoint indicator variable for the HPV detection types (i.e., PCR, ISH, immunohistochemistry, serology, and Southern blot) but no other covariates. Median values of HPV prevalence were also calculated (Supplementary Table 2). The multivariable model of HPV prevalence was adjusted for all study characteristics (i.e., HPV detection method, continent, publication date, and study size) (Table 2). For all models, study-level estimates of overall and typespecific HPV prevalence were meta-analysed using the metareg command in STATA to obtain random-effects summary estimates (DerSimonian and Laird, 1986; Thompson and Sharp, 1999).

In the crude models, we also estimated the among-population variance $\left(\tau^{2}\right)$. Tau-squared is the variance of the presumptively normal distribution of true values among populations in which each population has its own true value (i.e., its own true HPV prevalence). We utilised $\tau^{2}$ to calculate a $95 \%$ population effects interval (PEI) within which $95 \%$ of the populations' true values are estimated to lie (i.e., mean prevalence $\pm 1.96^{\star} \tau$ ) (Mosteller and Colditz, 1996; Ludema et al, 2011). To calculate the PEI, we used a logit transformation (Barendregt et al, 2013).

Two sensitivity analyses were performed to assess the robustness of the results. First, the analysis was performed excluding the PCR studies with beta-globin-negative cases (i.e., the studies where the total number of available OSCC cases differed from the number of OSCC cases actually tested for HPV) to assess the overall influence of these data on the estimates. Second, summary estimates of overall HPV prevalence were calculated for the serology studies, stratified by L1 and E6/E7 antigen status.

Meta-analyses were performed using STATA version 12 for Windows (StataCorp LP, College Station, TX, USA). Ancillary analyses were performed using SAS version 9.2 (SAS Institute, Inc., Cary, NC, USA).

\section{RESULTS}

Systematic review and study characteristics. Our systematic search of PubMed and EMBASE resulted in 724 unique papers. Of these, $124(17.1 \%)$ studies were eligible and included in this systematic review and meta-analysis. Of the remaining 600 publications, 585 (97.5\%) were excluded based on various exclusion criteria, $14(2.3 \%)$ were duplicate study populations, and $1(0.2 \%)$ was ineligible after contacting the author. The primary reasons for exclusion included the following: the outcome studied not being oesophageal cancer ( $n=185$ publications) and the paper being a review article or letter to the editor without original data ( $n=137$ publications) (Figure 1$)$.

Individual characteristics of the included 124 studies are summarised in Supplementary Table 1. Study sample sizes ranged from 20 to 1561 OSCC cases (median $=60$ ). Summing across studies, a total of 14047 OSCC cases were identified. However, as 215 case samples did not have sufficient DNA for analysis, a total of 13832 OSCC cases were included in the analysis. As reported in Table 1, the majority of studies were conducted in Asian countries $(n=77,62.1 \%)$, with the remaining studies spanning five other continents as follows: $20(16.1 \%)$ studies in Europe, 9 (7.3\%) studies in Africa, 8 (6.5\%) studies in North America, $4(3.2 \%)$ studies in South America, 1 (0.8\%) study in Australia, and $5(4.0 \%)$ studies included populations from more than one continent. The majority (70.9\%) of the Chinese studies were from the high-OSCC-incidence provinces of China. For multi-country studies, information was extracted separately by country for five multi-continent studies (Togawa et al, 1994; Suzuk et al, 1996; Wang et al, 2010; Castillo et al, 2011; Sitas et al, 2012) and for five single-continent studies (Mori et al, 1989; Turner et al, 1997; Kawaguchi et al, 2000; Castillo et al, 2006; Goto et al, 2011).

The majority of studies $(n=90,72.6 \%)$ used only one laboratory detection method to detect HPV, whereas 29 (23.4\%) studies used two detection methods and 5 (4.0\%) studies

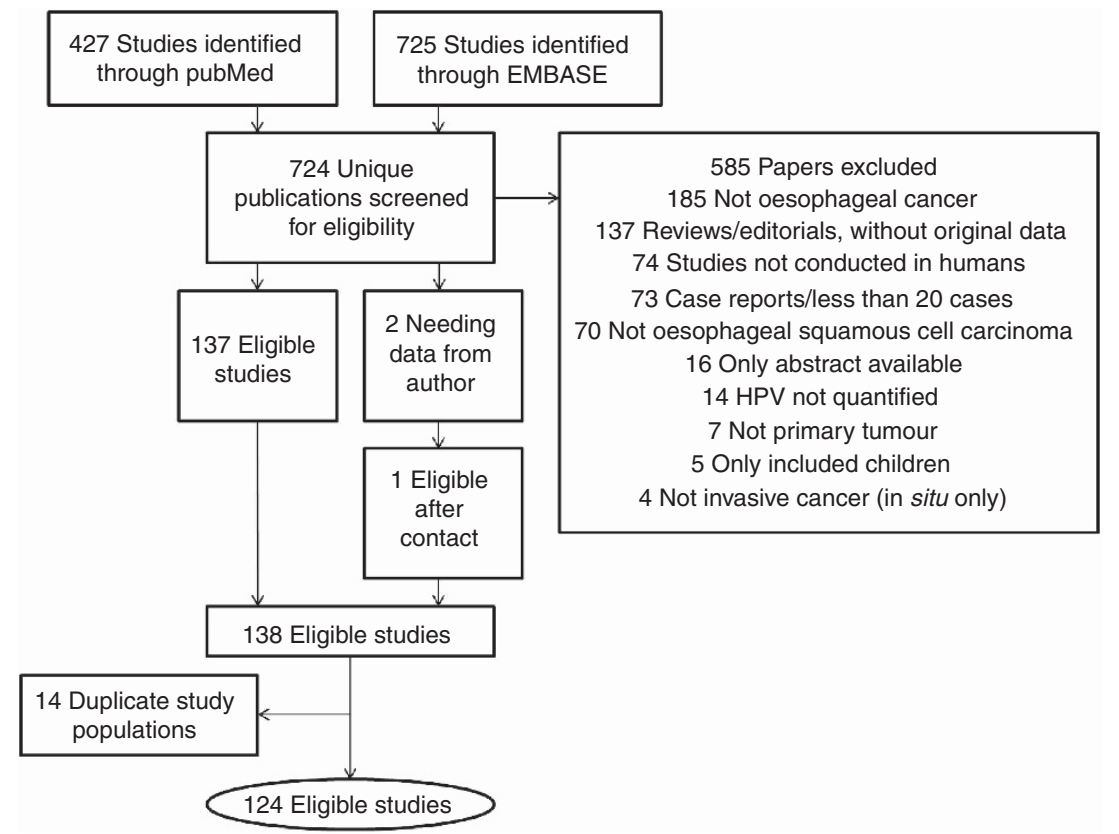

Figure 1. Flow diagram of inclusion and exclusion of identified studies for the meta-analysis of overall prevalence of human papillomavirus (HPV) among oesophageal squamous cell carcinoma (OSCC) cases. Although many articles were excluded based on multiple criteria, we assigned primary exclusion criteria according to the following rank order: study not conducted in humans (i.e., in vitro or animal study), not oesophageal cancer, not squamous cell carcinoma (histology known), not primary tumour, not invasive (i.e., in situ only), study population children only (i.e., $<18$ years old), letter to the editor or review (without original data), case report, abstract, histology unidentified, $<20$ cases, and no quantification of HPV. 
used three detection methods. The total number of estimates was 174 summing the across-study populations and detection methods. The majority of the estimates $(n=109,62.6 \%)$ were based on PCR methods (Table 2).
Meta-analysis of HPV prevalence in OSCC cases. The pooled prevalence estimates for studies with non-zero prevalence estimates, stratified by detection method, were 0.325 by PCR; 0.312 by ISH; 0.359 by immunohistochemistry; 0.321 by L1 serology; and 0.201

Table 1. Selected characteristics of 124 studies included in the meta-analysis of overall prevalence of human papillomavirus (HPV) among oesophageal squamous cell carcinoma (OSCC) cases

\begin{tabular}{|c|c|c|c|}
\hline Continent & Studies, $N$ & $\begin{array}{l}\text { OSCC } \\
\text { Cases, } N^{a}\end{array}$ & Countries represented \\
\hline Africa & 9 & 1235 & Egypt, Kenya, South Africa \\
\hline Asia & 77 & 9861 & China $^{\mathbf{b}}$, India, Iran, Japan, Pakistan, South Korea, Taiwan, Turkey \\
\hline Australia & 1 & 433 & Australia \\
\hline Europe & 20 & 1275 & $\begin{array}{l}\text { Belgium, Czech Republic, Finland, France, Germany, Greece, Hungary, Italy, Netherlands, Norway, Poland, } \\
\text { Romania, Russia, Slovenia, Sweden, United Kingdom }\end{array}$ \\
\hline North America & 8 & 280 & Canada, Mexico, United States \\
\hline South America & 4 & 748 & Brazil, Chile, Colombia \\
\hline Multi-continent & 5 & - & \\
\hline Total & 124 & 13832 & \\
\hline
\end{tabular}

Table 2. Multiple meta-regression of overall prevalence ${ }^{a}$ of human papillomavirus (HPV) among oesophageal squamous cell carcinoma (OSCC) cases

\begin{tabular}{|c|c|c|c|c|c|}
\hline Characteristic $^{\mathbf{b}}$ & $\begin{array}{l}\text { Studies } \\
N\end{array}$ & $\begin{array}{l}\text { Estimate } \\
N\end{array}$ & $\begin{array}{l}\text { OSCC } \\
\text { cases } N\end{array}$ & $\begin{array}{c}\text { Univariable } \\
\text { meta-regression model }{ }^{\mathrm{c}}\end{array}$ & $\begin{array}{c}\text { Multivariable } \\
\text { meta-egression model }\end{array}$ \\
\hline & & & & & Prevalence $(95 \% \mathrm{Cl})$ \\
\hline Intercept $^{\mathrm{e}}$ & & & & & $0.403(0.337,0.470)$ \\
\hline HPV detection method & & & & Prevalence $(95 \% \mathrm{Cl})$ & Prevalence Difference $(95 \% \mathrm{Cl})$ \\
\hline $\begin{array}{l}\text { PCR } \\
\text { In situ hybridisation } \\
\text { Immunohistochemistry } \\
\text { Serology } \\
\text { Southern blot }\end{array}$ & $\begin{array}{r}95 \\
25 \\
7 \\
14 \\
11\end{array}$ & $\begin{array}{r}109 \\
28 \\
7 \\
14 \\
16\end{array}$ & $\begin{array}{c}9257 \\
2631 \\
773 \\
862 \\
556\end{array}$ & $\begin{array}{l}0.277(0.234,0.320) \\
0.243(0.159,0.326) \\
0.304(0.185,0.423) \\
0.322(0.154,0.490) \\
0.176(0.061,0.292)\end{array}$ & $\begin{array}{c}\text { REF } \\
-0.034(-0.126,0.059) \\
0.067(-0.191,0.058) \\
0.112(-0.054,0.277) \\
-0.067(-0.191,0.058)\end{array}$ \\
\hline \multicolumn{6}{|l|}{ Continent } \\
\hline $\begin{array}{l}\text { Asia } \\
\text { Europe/Australia } \\
\text { North America } \\
\text { South America } \\
\text { Africa }\end{array}$ & $\begin{array}{r}84 \\
22 \\
8 \\
5 \\
10\end{array}$ & $\begin{array}{r}116 \\
27 \\
11 \\
8 \\
12\end{array}$ & $\begin{array}{c}10985 \\
1410 \\
350 \\
664 \\
670\end{array}$ & $\begin{array}{l}0.314(0.274,0.354) \\
0.156(0.073,0.238) \\
0.166(0.036,0.296) \\
0.194(0.042,0.346) \\
0.198(0.075,0.322)\end{array}$ & $\begin{array}{c}\text { REF } \\
-0.180(-0.277,-0.084) \\
-0.166(-0.304,-0.027) \\
-0.169(-0.327,-0.011) \\
-0.113(-0.244,0.018)\end{array}$ \\
\hline \multicolumn{6}{|l|}{ Publication date } \\
\hline $\begin{array}{l}2000 \text { to present } \\
1990-1999 \\
\text { Prior to } 1990\end{array}$ & $\begin{array}{r}83 \\
36 \\
3\end{array}$ & $\begin{array}{r}105 \\
65 \\
4\end{array}$ & $\begin{array}{c}10621 \\
3322 \\
136\end{array}$ & $\begin{array}{l}0.310(0.267,0.352) \\
0.204(0.149,0.259) \\
0.103(-0.114,0.321)\end{array}$ & $\begin{array}{c}\text { REF } \\
-0.093(-0.168,-0.017) \\
-0.282(-0.536,-0.029)\end{array}$ \\
\hline \multicolumn{6}{|l|}{ Study size } \\
\hline $\begin{array}{l}\leqslant 60 \text { OSCC cases } \\
>60 \text { OSCC cases }\end{array}$ & $\begin{array}{l}65 \\
63\end{array}$ & $\begin{array}{l}98 \\
76\end{array}$ & $\begin{array}{c}3410 \\
10669\end{array}$ & $\begin{array}{l}0.272(0.226,0.318) \\
0.261(0.211,0.311)\end{array}$ & $\begin{array}{c}\text { REF } \\
-0.092(-0.162,-0.023)\end{array}$ \\
\hline \multicolumn{6}{|c|}{$\begin{array}{l}\text { a Two studies that did not report overall HPV were excluded (Ma et al, 2000; Sitas et al, 2012). } \\
\text { b Categories are exclusive for estimates but not for studies because some studies presented results for multiple detection methods. } \\
\text { c Univariable meta-regression of a single study characteristic, where the HPV prevalence estimates are not controlled for other study characteristics. For example, the prevalence of } \\
\text { PCR is adjusted for other HPV detection types (i.e., in situ hybridisation, immunohistochemistry, serology and Southern blot) but no other study characteristics (i.e., continent, publication date, } \\
\text { or study size). } \\
\text { d }_{\text {Multivariable meta-regression, where the HPV prevalence estimates are controlled for all other study characteristics in the table. }} \\
\text { e Intercept: Asian PCR studies conducted } 2000 \text { to present with } \leqslant 60 \text { OSCC cases. }\end{array}$} \\
\hline
\end{tabular}


by Southern and other blotting techniques. We utilised the unrounded values of these prevalence estimates to smooth values for studies with zero HPV-positive cases.

The overall random-effects summary estimates from the crude model for HPV were similar across detection methods. The average prevalence of HPV (95\% confidence interval (CI)) was 0.278 (95\% CI: $0.233,0.322)$ by PCR; 0.242 (95\% CI: $0.157,0.328)$ by ISH; 0.305 (95\% CI: $0.160,0.450$ ) by immunohistochemistry; 0.321 (95\% CI: $0.156,0.486)$ by L1 serology; and 0.166 (95\% CI: 0.097 , 0.234 ) by Southern blot (Supplementary Table 2). Utilising the logit transformation, the average prevalence of $\mathrm{HPV}(95 \%$ population effects interval (PEI)) was 0.292 (95\% PEI: 0.039 , 0.809 ) by PCR; 0.280 (95\% PEI: $0.045,0.763$ ) by ISH; 0.329 (95\% PEI: $0.050,0.821)$ by immunohistochemistry; 0.302 (95\% PEI: $0.075,0.698$ ) by L1 serology; and 0.213 (95\% PEI: 0.084 , 0.442 ) by Southern blot (data not shown in tables). In general, the median per cent positive estimates were similar to the summary medians. The median prevalence of overall HPV positivity was 0.211 by PCR; 0.221 by ISH; 0.232 by immunohistochemistry; 0.275 by L 1 serology; and 0.180 by Southern blot (Supplementary Table 2).

In the univariable model, the mean HPV prevalence was 0.277 (95\% CI: $0.234,0.320)$ for PCR, 0.314 (95\% CI: 0.274, 0.354) for Asia, 0.310 (95\% CI: $0.267,0.352)$ for publications after 1999, and 0.272 (95\% CI: $0.226,0.318)$ for studies with $\leqslant 60$ cases. In the multivariable meta-regression analysis, the mean HPV prevalence for the intercept (Asian PCR studies conducted after 1999 with $\leqslant 60$ cases) was 0.403 ( $95 \%$ CI: $0.337,0.470)$. By detection method, estimates for L1 serology were the most different from PCR, with a prevalence difference of 0.112 higher than the intercept. Among continents, Europe/Australia was the most different from Asia, with a prevalence difference of -0.180 . Studies conducted prior to 1990 were the most different from studies conducted from 2000 to present, with a prevalence difference of -0.282 . Finally, studies with $>60$ cases had a prevalence difference of -0.092 compared with studies with $\leqslant 60$ cases (Table 2 ).

The range for HPV prevalence was $0-84.1 \%$ for PCR; $0-72.3 \%$ for ISH; $0-73.3 \%$ for immunohistochemistry; $3.2-58.5 \%$ for serology; and $0-45.0 \%$ for Southern/slot/dot blot (Supplementary Table 1). The prevalence of high-risk types of HPV, namely HPV-16 and -18, was fairly similar across PCR, ISH, and Southern blot, but differed slightly for serology and immunohistochemistry (Supplementary Table 2). HPV-16 prevalence was 0.185 (95\% CI: $0.142,0.228)$ for PCR; 0.255 (95\% CI: 0.114, 0.397) for ISH; 0.359 (95\% CI: - 0.101, 0.819 ) for immunohistochemistry; 0.173 (95\% CI: $0.087,0.259)$ for L1 serology; and 0.187 (95\% CI: $0.116,0.259$ ) for Southern blot. Median prevalence estimates for HPV-16 were 0.132 for PCR; 0.190 for ISH; 0.430 for immunohistochemistry; 0.084 for L1 serology; and 0.198 for Southern blot.

Results stratified by detection method for HPV prevalence according to continent, publication year, DNA source, and primer set (for PCR only) are provided in Supplementary Table 2. As with the meta-regression, striking differences were noted for study location across HPV detection methods. Trends across publication year, tissue source, and primer set were noted but were not as consistent across detection methods (Supplementary Table 2).

We also examined within-study comparison for studies that had both PCR and ISH $(n=11)$, PCR and Southern blot $(n=9)$, PCR and immunohistochemistry $(n=3)$, ISH and immunohistochemistry $(n=4)$, ISH and Southern blot $(n=3)$, and HPV 16 and 18 $(n=62)$. The results from these analyses were similar to our overall results. For example, the within-study meta-analysis for ISH compared with PCR resulted in a prevalence difference of -0.038 (95\% CI: $-0.024,0.164)$, based on a mean prevalence of 0.202 (95\% CI: $0.059,0.345)$ for PCR (data not shown in tables).

In our sensitivity analyses, results were similar when excluding the PCR studies with beta-globin-negative cases. When the PCR analysis is restricted to only studies with all beta-globin-positive cases, the overall HPV prevalence is 0.288 (95\% CI: 0.238, 0.338). However, results were dissimilar for serology studies when stratified by L1 or E6/E7 antigen status. For example, the overall HPV prevalence for E6/E7 antigen status studies was 0.100 (95\% CI: $-0.777,0.978)$. These results were based on only two studies that utilised E6/E7 antigen status (Dai et al, 2007; Guo et al, 2012). Therefore, only L1 serology results are presented in the tables.

\section{DISCUSSION}

This systematic review and meta-analysis of the literature on HPV prevalence combined data on 13832 OSCC cases from 124 studies. In our study, we utilised multivariable meta-regression methods to analyse HPV prevalence in OSCC tumours. We summarised the influence of several important factors on HPV prevalence in OSCC cases, including HPV detection method, geographic location, publication date, and study size.

The prevalence of overall HPV varied widely across detection methods, ranging from $17.6 \%$ for Southern blot to $32.2 \%$ for $\mathrm{L} 1$ serology. Our assessment of heterogeneity suggested meaningful variation in HPV prevalence across HPV detection methods. The estimates of overall HPV prevalence from our meta-analysis are consistent with a recent meta-analysis by Syrjanen, which reported an overall summary HPV prevalence of $29.0 \%$ (95\% CI: 25.1-33.1\%), after combining data on histological biopsies, dot blot hybridisation, filter ISH, hybrid capture 2 assay, ISH, PCR, and Southern blot hybridisation (Syrjanen, 2013). In contrast, we have only presented stratum-specific estimates by detection method in order to elucidate the heterogeneity in HPV prevalence estimates. Each detection method has strengths and limitations with regard to measurement of possible infection at different time points, technical requirements, and test characteristics. For instance, PCR, Southern blot, and ISH have high specificity, ISH has low sensitivity, and PCR has a high rate of false positives (Kimple et al, 2012).

Further, it is uninformative to estimate an overall (i.e., worldwide) HPV prevalence, as it is a function of the number of HPV types that are tested and a host of other methodologic determinants as well as highly variable characteristics of person, place, and time. Testing for additional HPV types could partially account for the increasing prevalence across time. However, this seems unlikely as HPV-16 is the predominant type detected and is nearly always tested for as a high-risk HPV type.

The observed differences in HPV prevalence by geographic location warrant further attention. Most notably, our study uniquely reports estimates for overall HPV prevalence in Chinese regions characterised by the high incidence of OSCC. Our estimates of HPV prevalence in these Chinese regions, ranging from $26.4 \%$ (95\% CI: $10.8 \%, 42.0 \%$ ) for Southern blot to $41.6 \%$ (95\% CI: $33.1 \%, 50.2 \%$ ) for PCR, are substantially higher than any continent-specific estimates, including other regions of Asia. Interestingly, all of our detection method-specific estimates for high-risk provinces of China are lower than the estimate of $41.8 \%$ (95\% CI: 34.3\%, 49.7\%) reported by Syrjanen (2013) for Chinese studies, which includes both high- and low-risk Chinese regions. This is possible due to the inclusion of additional detection types not included in our analysis (e.g., histological biopsies, hybrid capture 2 assay). In a continent-specific analysis stratified by the HPV detection method, we found that HPV prevalence was highest in Asia and Africa and similar across all other continents. These results are consistent with (1) the estimates reported by Syrjanen that show high prevalence in China and South Africa (Syrjanen, 2013) and (2) a systematic review by Liyanage et al (2013) that reported higher levels of HPV detection in regions with high OSCC risk. 
Although the geographic differences in oral HPV prevalence are notable (Kreimer et al, 2010), HPV cannot be expected to fully account for the geographical variation seen in OSCC incidence (Ferlay et al, 2013). Even when an infectious agent participates in the aetiology of every case of a disease (e.g., tubercle bacillus and tuberculosis, Plasmodium protozoa and malaria, or HIV and AIDS), aetiologic co-factors help determine the disease's geographic variation.

Further, these findings need to be cautiously interpreted, as they do not account for the background prevalence of HPV. Future studies need to account for the background prevalence of HPV, which varies widely by region. A recent meta-analysis examined the prevalence of oral HPV, ranging from approximately $2.6 \%$ in Canada to $18.9 \%$ in Brazil (Kreimer et al, 2010). Additionally, this review cannot account for other established risk factors, including tobacco, alcohol, poverty, and nutritional deficiencies (IARC, 1986; IARC, 1988; Munoz and Castellsague, 1994; Hongo et al, 2009; Mao et al, 2011).

HPV-16 was the predominant type of HPV assayed and identified across detection methods, with the exception of L1 serology. In stratified analyses, HPV-16 prevalence ranged from $17.3 \%$ for L1 serology to $35.9 \%$ for immunohistochemistry. HPV18 was the predominate type of HPV identified for L1 serology, with a prevalence of $26.0 \%$. HPV-18 prevalence ranged from $0 \%$ for immunohistochemistry to $26.0 \%$ for L1 serology. Our results for PCR were consistent with a recent meta-analysis by Yong et al (2013), which reported a HPV-16 prevalence of $11.7 \%$ (95\% CI: $7.7 \%, 16.2 \%)$ and a HPV-18 prevalence of $1.8 \%$ (95\% CI: $0.9 \%$, $3.0 \%$ ) in OSCC tumours detected with PCR alone. Unlike previous studies, our analysis also summarises the differences in HPV typespecific prevalence across multiple detection methods.

Characterising the prevalence of HPV in OSCC cases is an important preliminary step to assessing the association between HPV and OSCC. The prevalence of HPV among OSCC cases in this meta-analysis is lower than the prevalence of HPV previously observed for other cancers with more established epidemiologic evidence for HPV involvement, such as cervical, anal, oropharyngeal, or tonsillar (IARC, 2012). Nonetheless, the HPV prevalence in OSCC cases is high enough, and correlated strongly enough with OSCC incidence ecologically, suggesting that an aetiologic connection might be present between the virus and the cancer. By way of analogy, we note that the prevalence of cigarette smoking is lower in bladder cancer cases than in cases of lung cancer. A causal connection between smoking and bladder cancer was established nonetheless. The requisite research took longer, largely because smoking's association with bladder cancer, though causal, was weaker than its association with lung cancer.

An association between HPV and OSCC is biologically plausible on the basis of (1) the close proximity and histologic similarities between oesophageal and oral squamous epithelium - suggesting that the oesophagus could be exposed to HPV in a similar fashion as the oral cavity and oropharynx (Gillison and Shah, 2003); (2) the detection of HPV DNA in both benign oesophageal squamous cell papillomas and malignant OSCC tumour samples (Syrjanen, 2002); (3) in vivo studies in cattle, showing that bovine papillomavirus leads to the development of papillomas, then to squamous cell carcinoma (Borzacchiello and Roperto, 2008); and (4) in vitro experiments of human oesophageal epithelial cells where HPV-18 was shown to be an initiating factor in cancer transformation (Shen et al, 2000). However, counterarguments have been suggested, including the lack of association between OSCC and immunosuppression or previous HPV-associated disease (Gillison and Shah, 2003) and wide variability in case-series report of HPV positivity in OSCC cases (Syrjanen, 2002) - ranging worldwide from over 70\% positivity (Liu et al, 2000; Cao et al, 2005; Zhu et al, 2005; Yang et al, 2008; Ding et al, 2009; Qu et al, 2012) to 0\% (Hale et al, 1989; Loke et al, 1990; Benamouzig et al, 1995; Smits et al,
1995; Saegusa, 1997; Morgan et al, 1997; Kamath et al, 2000; Talamini et al, 2000; Awerkiew et al, 2003; White et al, 2005; Koh et al, 2008; Bellizzi et al, 2009; Song et al, 2009; Patel et al, 2011; Noori et al, 2012; Haeri et al, 2013). Even within countries, the prevalence of HPV varies widely. For example, in one Chinese study, HPV positivity in OSCC tumours has been reported to range from $2.0 \%$ in Linxian province to $22.2 \%$ in Shantou province (Si et al, 2003). This variation is unlikely due to differences in the study methodology. The large variation in HPV DNA prevalence estimates in OSCC tumours across geographical sites has not been fully explained, but it could possibly be due to small sample sizes, differing HPV detection methods and PCR primers, cut points utilised for HPV positivity, variability between laboratories, and contamination of samples (Hubbard, 2003; Koshiol et al, 2010).

Contamination of samples is of paramount concern, as studies have reported that dehydrated HPV maintains 100\% infectivity for as long as a day (Roden et al, 1997). Additional studies have found HPV DNA on fomites and various medical surfaces (Ferenczy et al, 1989; Strauss et al, 2002). This is problematic because HPV DNA is resistant to cleaning detergents (Strauss et al, 2003), which leads to concerns that fomites could be responsible for contaminating samples (Roden et al, 1997). Although we attempted to evaluate quality control measures, this was difficult as many studies do not report on these issues, and we were concerned about biasing our results by including only studies that report on quality control measures. Future studies should strive to carefully avoid contamination and to report quality control measures.

Limitations of the current meta-analysis should be considered. Bias could arise from the study estimates because the accuracy of these estimates depends on the detection method utilised and the types of HPV assessed. That is, some studies utilised broad primers or multiple probes to detect multiple types of HPV, whereas other studies only detected a specific HPV type (e.g., HPV-16). Additionally, there is potential publication bias. This potential is seen in the multivariable meta-regression, which suggests that smaller studies may have higher reported prevalence. Finally, we used a continent-level variable to categorise studies, in addition to high-risk China and Iran. This classification may have masked important variability between studies on the same continent.

The studies included in this meta-analysis are heterogeneous. We directly examined heterogeneity by describing the prevalence of HPV in OSCC cases by HPV type, continent, publication date, DNA source, and primer set. Of course, we were not fully able to explain the heterogeneity. Prevalence estimates remained heterogeneous even in stratified results (e.g., PCR studies conducted in Asia). This is to be expected, as a full explanation of heterogeneity would require knowledge of every determinant of HPV prevalence in OSCC cases, as well as every determinant of HPV measurement validity and reliability, and a sufficient number of studies to explore possible combinations of all such variables. Nonetheless, we identified several variables that account for substantial portions of the heterogeneity.

Strengths of the current meta-analysis include large overall sample size, use of multivariable meta-regression to assess study characteristics, examination of type-specific HPV prevalence, inclusion of studies published in all languages, and exclusion of case reports or studies with small sample size. The use of multivariable meta-regression allowed us to determine study characteristics that influence heterogeneity of HPV prevalence estimates. By examining type-specific HPV prevalence, we were able to determine what is the predominate type of HPV detected in OSCC cases. However, we are still dependent on the HPV types that are included in the primer sets, which are largely optimised for the detection of HPV genital types. By including studies of all languages, we avoided selection bias due to publication language. Finally, by excluding case reports and studies with $<20$ cases, we 
attempted to exclude studies that were a convenience sample or not representative of all OSCC cases.

Although a high proportion of OSCC cases with prevalent HPV does not determine aetiologic significance, the data presented in this paper strongly indicate that such a hypothesis warrants further investigation. Similar to that which has been done for head and neck cancer (Agrawal et al, 2011), advances in aetiologic understanding of the role of HPV in OSCC tumour development will come from exome sequencing, which will ideally be conducted in a large number of tumours from differing geographic locals and differing tumour sites.

In summary, our results suggest that HPV prevalence in OSCC tumours is highest in Africa and Asia, especially in the regions of China with a high incidence of OSCC, and that high-risk HPV-16 is the most commonly identified HPV type in OSCC tumours. Our meta-analysis demonstrates that HPV detection method, continent, publication date, and study size contribute to the variability among individual study findings. Utilising multivariable metaregression, this systematic review highlights study characteristics that need to be considered when examining the association between HPV infection and OSCC. Although this review is a preliminary step in assessing the relationship between HPV and OSCC, it may also be used to better tailor monitoring and public health interventions (e.g., HPV vaccinations) if that relationship becomes established as causal. Future research should focus on quantifying $\mathrm{HPV}$ in OSCC cases using strict quality control measures to avoid contamination, as well as determining the association between HPV and OSCC incidence by conducting large, population-based case-control studies. Such studies will provide a richer understanding of the role of HPV in OSCC aetiology.

\section{ACKNOWLEDGEMENTS}

We are grateful to Anne Rositch for her advice on meta-analytic methods and Hazel Nichols for her feedback on the manuscript. Additionally, we are grateful to Hilda Razzaghi and Csaba Marosvari for their help in translating a Persian and Hungarian article, respectively. We would also like to acknowledge the efforts of the contacted study authors for providing additional data to this meta-analysis. This was supported by the National Institutes of Health (NIH), National Cancer Institute (T32CA009330-25) and the National Institutes of Environmental Health and Susceptibility (T32ES007018), as well as in part by the Intramural Research Programme of the NIH, National Institute of Environmental Health Sciences.

\section{REFERENCES}

Agrawal N, Frederick MJ, Pickering CR, Bettegowda C, Chang K, Li RJ, Fakhry C, Xie TX, Zhang J, Wang J, Zhang N, El-Naggar AK, Jasser SA, Weinstein JN, Trevino L, Drummond JA, Muzny DM, Wu Y, Wood LD, Hruban RH, Westra WH, Koch WM, Califano JA, Gibbs RA, Sidransky D, Vogelstein B, Velculescu VE, Papadopoulos N, Wheeler DA, Kinzler KW, Myers JN (2011) Exome sequencing of head and neck squamous cell carcinoma reveals inactivating mutations in NOTCH1. Science 333(6046): 1154-1157.

Awerkiew S, Bollschweiler E, Metzger R, Schneider PM, Holscher AH, Pfister H (2003) Esophageal cancer in germany is associated with EpsteinBarr-virus but not with papillomaviruses. Med Microbiol and Immunol 192(3): 137-140.

Barendregt JJ, Doi SA, Lee YY, Norman RE, Vos T (2013) Meta-analysis of prevalence. J Epidemiol Community Health 67(11): 974-978.

Bellizzi AM, Woodford RL, Moskaluk CA, Jones DR, Kozower BD, Stelow EB (2009) Basaloid squamous cell carcinoma of the esophagus: Assessment for high-risk human papillomavirus and related molecular markers. Am J Surg Pathol 33(11): 1608-1614.

Benamouzig R, Jullian E, Chang F, Robaskiewicz M, Flejou JF, Raoul JL, Coste T, Couturier D, Pompidou A, Rautureau J (1995) Absence of human papillomavirus DNA detected by polymerase chain reaction in French patients with esophageal carcinoma. Gastroenterology 109(6): 1876-1881.

Borzacchiello G, Roperto F (2008) Bovine papillomaviruses, papillomas and cancer in cattle. Vet Res 39(5): 45.

Cao B, Tian X, Li Y, Jiang P, Ning T, Xing H, Zhao Y, Zhang C, Shi X, Chen D, Shen Y, Ke Y (2005) LMP7/TAP2 gene polymorphisms and HPV infection in esophageal carcinoma patients from a high incidence area in China. Carcinogenesis 26(7): 1280-1284.

Castellsague X, Munoz N, De Stefani E, Victora CG, Castelletto R, Rolon PA, Quintana MJ (1999) Independent and joint effects of tobacco smoking and alcohol drinking on the risk of esophageal cancer in men and women. Int J Cancer 82(5): 657-664.

Castillo A, Aguayo F, Koriyama C, Torres M, Carrascal E, Corvalan A, Roblero JP, Naquira C, Palma M, Backhouse C, Argandona J, Itoh T, Shuyama K, Eizuru Y, Akiba S (2006) Human papillomavirus in esophageal squamous cell carcinoma in Colombia and Chile. World J Gastroenterol 12(38): 6188-6192.

Castillo A, Koriyama C, Higashi M, Anwar M, Bukhari MH, Carrascal E, Mancilla L, Okumura H, Matsumoto M, Sugihara K, Natsugoe S, Eizuru Y, Akiba S (2011) Human papillomavirus in upper digestive tract tumors from three countries. World J Gastroenterol 17(48): 5295-5304.

Chen W, He Y, Zheng R, Zhang S, Zeng H, Zou X, He J (2013) Esophageal cancer incidence and mortality in China, 2009. J Thorac Dis 5(1): 19-26.

Clifford GM, Smith JS, Plummer M, Munoz N, Franceschi S (2003) Human papillomavirus types in invasive cervical cancer worldwide: a meta-analysis. Br J Cancer 88(1): 63-73.

Corley DA, Buffler PA (2001) Oesophageal and gastric cardia adenocarcinomas: analysis of regional variation using the Cancer Incidence in Five Continents database. Int J Epidemiol 30(6): 1415-1425.

Dai M, Zhang WD, Clifford GM, Gheit T, He BC, Michael KM, Waterboer T, Hainaut P, Tommasino M, Franceschi S (2007) Human papillomavirus infection among 100 oesophageal cancer cases in the People's Republic of China. Int J Cancer 121(6): 1396-1398.

DerSimonian R, Laird N (1986) Meta-analysis in clinical trials. Control Clin Trials 7(3): 177-188.

Ding GC, Wang LD, Li SH, Feng CW, Zhang YR, Ren JL, Fan ZM, Wang R, He X, Guo T (2009) Correlation between human papillomavirus infection and esophageal squamous cell carcinoma and gastric cardia adenocarcinoma in high-incidence area of Henan province. Chin Cancer PrevTreat 16(4): 252-255.

Ferenczy A, Bergeron C, Richart RM (1989) Human papillomavirus DNA in fomites on objects used for the management of patients with genital human papillomavirus infections. Obstet Gynecol 74(6): 950-954.

Ferlay J, Shin HR, Bray F, Forman D, Mathers C, Parkin DM (2010) GLOBOCAN 2008 v1.2, Cancer Incidence and Mortality Worldwide: IARC CancerBase No. 10 [Internet]. International Agency for Research on Cancer: Lyon, France.

Ferlay J, Soerjomataram I, Ervik M, Dikshit R, Eser S, Mathers C, Rebelo M, Parkin DM, Forman D, Bray F (2013) GLOBOCAN 2012 v1.0, Cancer Incidence and Mortality Worldwide: IARC CancerBase No. 11 [Internet]. International Agency for Research on Cancer: Lyon, France.

Gillison ML, Shah KV (2003) Chapter 9: Role of mucosal human papillomavirus in nongenital cancers. J Natl Cancer Inst Monogr 31: 57-65.

Glenn TF (2001) Esophageal cancer. Facts, figures, and screening. Gastroenterol Nurs 24(6): 271-273quiz 274-5.

Goto A, Li CP, Ota S, Niki T, Ohtsuki Y, Kitajima S, Yonezawa S, Koriyama C, Akiba S, Uchima H, Lin YM, Yeh KT, Koh JS, Kim CW, Kwon KY, Nga ME, Fukayama M (2011) Human papillomavirus infection in lung and oesophageal cancers: analysis of 485 Asian cases. J Med Virol 83(8): 1383-1390.

Guo F, Liu Y, Wang X, He Z, Weiss NS, Madeleine MM, Liu F, Tian X, Song Y, Pan Y, Ning T, Yang H, Shi X, Lu C, Cai H, Ke Y (2012) Human papillomavirus infection and esophageal squamous cell carcinoma: a case-control study. Cancer Epidemiol Biomark Prev 21(5): 780-785.

Haeri H, Mardany O, Asadi-Amoli F, Shahsiah R (2013) Human papilloma virus and esophageal squamous cell carcinoma. Acta Med Iran 51(4): 242-245.

Hale MJ, Liptz TR, Paterson AC (1989) Association between human papillomavirus and carcinoma of the oesophagus in South African blacks. 
A histochemical and immunohistochemical study. S Afr Med J 76(7): 329-330.

Hongo M, Nagasaki Y, Shoji T (2009) Epidemiology of esophageal cancer: orient to occident. Effects of chronology, geography and ethnicity. J Gastroenterol Hepatol 24(5): 729-735.

Hubbard RA (2003) Human papillomavirus testing methods. Arch Pathol Lab Med 127(8): 940-945.

IARC (1986) IARC Monographs on the Evaluation of the Carcinogenic Risk of Chemicals to Humans: Tobacco Smoking. Vol. 38. International Agency for Research on Cancer: Lyon, France.

IARC (1988) IARC Monographs on the Evaluation of Carcinogenic Risks to Hhumans: Alcohol Drinking. Vol. 44. International Agency for Research on Cancer: Lyon, France.

IARC (2012) IARC Monographs on the Evaluation of Carcinogenic Risks to Humans: A Review of Human Carcinogens: Biological Agents. Vol. 100B. International Agency for Cancer Research: Lyon, France.

Kamangar F, Malekzadeh R, Dawsey SM, Saidi F (2007) Esophageal cancer in Northeastern Iran: a review. Arch Iran Med 10(1): 70-82.

Kamath AM, Wu TT, Heitmiller R, Daniel R, Shah KV (2000) Investigation of the association of esophageal carcinoma with human papillomaviruses. Dis Esophagus 13(2): 122-124.

Kawaguchi H, Ohno S, Araki K, Miyazaki M, Saeki H, Watanabe M, Tanaka S, Sugimachi K (2000) p53 polymorphism in human papillomavirusassociated esophageal cancer. Cancer Res 60(11): 2753-2755.

Kimple AJ, Torres AD, Yang RZ, Kimple RJ (2012) HPV-associated head and neck cancer: molecular and nano-scale markers for prognosis and therapeutic stratification. Sensors (Basel) 12(4): 5159-5169.

Koh JS, Lee SS, Baek HJ, Kim YI (2008) No association of high-risk human papillomavirus with esophageal squamous cell carcinomas among Koreans, as determined by polymerase chain reaction. Dis Esophagus 21(2): 114-117.

Koshiol J, Wei WQ, Kreimer AR, Chen W, Gravitt P, Ren JS, Abnet CC Wang JB, Kamangar F, Lin DM, Von Knebel-Doeberitz M, Zhang Y, Viscidi R, Wang GQ, Gillison ML, Roth MJ, Dong ZW, Kim E, Taylor PR, Qiao YL, Dawsey SM (2010) No role for human papillomavirus in esophageal squamous cell carcinoma in China. Int J Cancer 127(1): 93-100.

Kreimer AR, Bhatia RK, Messeguer AL, Gonzalez P, Herrero R, Giuliano AR (2010) Oral human papillomavirus in healthy individuals: a systematic review of the literature. Sex Transm Dis 37(6): 386-391.

Liu J, Su Q, Zhang W (2000) Relationship between HPV-E6 P53 protein and esophageal squamous cell carcinoma. World Chin J Digestol 8(5): 494-496.

Liyanage SS, Segelov E, Garland SM, Tabrizi SN, Seale H, Crowe PJ, Dwyer DE, Barbour A, Newall AT, Malik A, Macintyre CR (2013) Role of human papillomaviruses in esophageal squamous cell carcinoma. Asia Pac J Clin Oncol 9(1): 12-28.

Loke SL, Ma L, Wong M, Srivastava G, Lo I, Bird CC (1990) Human papillomavirus in oesophageal squamous cell carcinoma. J Clin Pathol 43(11): 909-912.

Ludema C, Cole SR, Poole C, Chu H, Eron JJ (2011) Meta-analysis of randomized trials on the association of prophylactic acyclovir and HIV-1 viral load in individuals coinfected with herpes simplex virus-2. AIDS 25(10): 1265-1269.

Ma QF, Jiang H, Feng YQ, Wang XP, Zhou YA, Liu K, Jia ZL (2000) Detection of human papillomavirus DNA in squamous cell carcinoma of the esophagus. World Chin J Digestol 8(11): 1218-1224.

Mao WM, Zheng WH, Ling ZQ (2011) Epidemiologic risk factors for esophageal cancer development. Asian Pac J Cancer Prev 12(10): 2461-2466.

Moher D, Liberati A, Tetzlaff J, Altman DG (2009) Preferred reporting items for systematic reviews and meta-analyses: the PRISMA statement. J Clin Epidemiol 62(10): 1006-1012.

Morgan RJ, Perry ACF, Newcomb PV, Hardwick RH, Alderson D (1997) Human papillomavirus and oesophageal squamous cell carcinoma in the UK. Eur J Surg Oncol 23(6): 513-517.

Mori M, Shimono R, Inoue T, Kuwano H, Sugimachi K, Zhang RG (1989) Papillomavirus and esophageal cancer in the Japanese and Chinese. Am J Gastroenterol 84(9): 1126-1127.

Mosteller F, Colditz GA (1996) Understanding research synthesis (metaanalysis). Annu Rev Public Health 17: 1-23.

Munoz N, Castellsague X (1994) Epidemiology of oesophageal cancer. Eur J Gastroenterol Hepatol 6(8): 649-655.
Noori S, Monabati A, Ghaderi A (2012) The prevalence of human papilloma virus in esophageal squamous cell carcinoma. Iran J Med Sci 37(2): $126-133$.

Patel K, Mining S, Wakhisi J, Gheit T, Tommasino M, Martel-Planche G, Hainaut P, Abedi-Ardekani B (2011) TP53 mutations, human papilloma virus DNA and inflammation markers in esophageal squamous cell carcinoma from the Rift Valley, a high-incidence area in Kenya. BMC Res Notes 4: 469.

Qu P, Li JT, Wang LD, Zeng Y, Chu XS (2012) [Comparative study on the HPV infection rate of different esophageal squamous cell carcinoma in Anyang China]. Zhonghua shi yan he lin chuang bing du xue za zhi 26(1): $34-36$.

Roden RB, Lowy DR, Schiller JT (1997) Papillomavirus is resistant to desiccation. J Infect Dis 176(4): 1076-1079.

Saegusa M (1997) Absence of human papillomavirus genomic sequences detected by the polymerase chain reaction in oesophageal and gastric carcinomas in Japan. Mol Pathol 50(2): 101-104.

Shen Z, Cen S, Shen J, Cai W, Xu J, Teng Z, Hu Z, Zeng Y (2000) Study of immortalization and malignant transformation of human embryonic esophageal epithelial cells induced by HPV18 E6E7. J Cancer Res Clin Oncol 126(10): 589-594.

Si HX, Tsao SW, Poon CSP, Wang LD, Wong YC, Cheung ALM (2003) Viral load of HPV in esophageal squamous cell carcinoma. Int J Cancer 103(4): 496-500.

Sitas F, Egger S, Urban MI, Taylor PR, Abnet CC, Boffetta P, O'Connell DL, Whiteman DC, Brennan P, Malekzadeh R, Pawlita M, Dawsey SM, Waterboer T, Webb PM, Green AC, Hayward NK, Zaridze D, Holcatova I, Mates D, Szeszenia-Dabrowska N, Ferro G, Janout V, Curado MP, Menezes AM, Koifman S, Islami F, Nasrollahzadeh D, Hu N, Goldstein AM, Gao Y, Ding T, Kamangar F (2012) InterSCOPE study: associations between esophageal squamous cell carcinoma and human papillomavirus serological markers. J Natl Cancer Inst 104(2): 147-158.

Smits HL, Tjong-Hung ASP, Ter Schegget J, Nooter K, Kok T (1995) Absence of human papillomavirus DNA from esophageal carcinoma as determined by multiple broad spectrum polymerase chain reactions. J Med Virol 46(3): 213-215.

Song CS, Cui JH, Wu ZP, Yang G, Tan JJ (2009) Relationship between detection of human papilloma virus genotypes and esophageal squamous cell carcinoma. Chin J Cancer Prev Treat 16(5): 329-331.

Strauss S, Sastry P, Sonnex C, Edwards S, Gray J (2002) Contamination of environmental surfaces by genital human papillomaviruses. Sex Transm Infect 78(2): 135-138.

Strauss S, Stephen H, Sonnex C, Gray J (2003) Contamination of environmental surfaces by genital human papillomaviruses (HPV): a follow up study. Sex Transm Infect 79(5): 426-427.

Suzuk L, Noffsinger AE, Hui YZ, Fenoglio-Preiser CM (1996) Detection of human papillomavirus in esophageal squamous cell carcinoma. Cancer 78(4): 704-710.

Sweeting MJ, Sutton AJ, Lambert PC (2004) What to add to nothing? Use and avoidance of continuity corrections in meta-analysis of sparse data. Stat Med 23(9): 1351-1375.

Syrjanen K (2013) Geographic origin is a significant determinant of human papillomavirus prevalence in oesophageal squamous cell carcinoma: systematic review and meta-analysis. Scand J Infect Dis 45(1): 1-18.

Syrjanen KJ (1982) Histological changes identical to those of condylomatous lesions found in esophageal squamous cell carcinomas. Arch Geschwulstforsch 52(4): 283-292.

Syrjanen KJ (2002) HPV infections and oesophageal cancer. J Clin Pathol 55(10): 721-728.

Talamini G, Capelli P, Zamboni G, Mastromauro M, Pasetto M, Castagnini A, Angelini G, Bassi C, Scarpa A (2000) Alcohol, smoking and papillomavirus infection as risk factors for esophageal squamous-cell papilloma and esophageal squamous-cell carcinoma in Italy. Int J Cancer 86(6): 874-878.

Thompson SG, Sharp SJ (1999) Explaining heterogeneity in meta-analysis: a comparison of methods. Stat Med 18(20): 2693-2708.

Togawa K, Jaskiewicz K, Takahashi H, Meltzer SJ, Rustgi AK (1994) Human papillomavirus DNA sequences in esophagus squamous cell carcinoma. Gastroenterology 107(1): 128-136.

Turner JR, Shen LH, Crum CP, Dean PJ, Odze RD (1997) Low prevalence of human papillomavirus infection in esophageal squamous cell carcinomas from North America: analysis by a highly sensitive and 
specific polymerase chain reaction-based approach. Human Pathol 28(2): $174-178$.

Wang X, Tian X, Liu F, Zhao Y, Sun M, Chen D, Lu C, Wang Z, Shi X, Zhang Q, Zhang D, Shen Z, Li F, Harris CC, Cai H, Ke Y (2010) Detection of HPV DNA in esophageal cancer specimens from different regions and ethnic groups: a descriptive study. BMC Cancer 10: 19.

Wei WQ, Yang J, Zhang SW, Chen WQ, Qiao YL (2011) Esophageal cancer mortality trends during the last 30 years in high risk areas in China: comparison of results from national death surveys conducted in the 1970s, 1990s and 2004-2005. Asian Pac J Cancer Prev 12(7): $1821-1826$.

White RE, Mungatana C, Mutuma G, Robert ME, Daniel RW, Topazian MD, Shah KV (2005) Absence of human papillomavirus in esophageal carcinomas from southwestern Kenya. Dis Esophagus 18(1): 28-30.

Yang W, Zhang Y, Tian X, Ning T, Ke Y (2008) p53 codon 72 polymorphism and the risk of esophageal squamous cell carcinoma. Mol Carcinog 47(2): $100-104$.
Yong F, Xudong N, Lijie T (2013) Human papillomavirus types 16 and 18 in esophagus squamous cell carcinoma: a meta-analysis. Ann Epidemiol 23(11): 726-734.

Yu Y, Taylor PR, Li JY, Dawsey SM, Wang GQ, Guo WD, Wang W, Liu BQ, Blot WJ, Shen Q et al. (1993) Retrospective cohort study of risk-factors for esophageal cancer in Linxian, People's Republic of China. Cancer Causes Control 4(3): 195-202.

Zhu LZ, Su XL, Chen KN, Yang RJ, Xing HP, Cui JG, Ke Y (2005) Detection rate of human papillomavirus-16 in esophageal squamous cell carcinoma from different Chinese populations. Ai zheng 24(7): 870-873.

This work is published under the standard license to publish agreement. After 12 months the work will become freely available and the license terms will switch to a Creative Commons AttributionNonCommercial-Share Alike 3.0 Unported License.

Supplementary Information accompanies this paper on British Journal of Cancer website (http://www.nature.com/bjc) 